\title{
Multivariate joint modeling to identify markers of growth and lung function decline that predict cystic fibrosis pulmonary exacerbation onset
}

\author{
E. R. Andrinopoulou', J. P. Clancy $2,3,4$ and R. D. Szczesniak $3,4,5^{*}$ (1)
}

\begin{abstract}
Background: Attenuated decreases in lung function can signal the onset of acute respiratory events known as pulmonary exacerbations (PEs) in children and adolescents with cystic fibrosis (CF). Univariate joint modeling facilitates dynamic risk prediction of PE onset and accounts for measurement error of the lung function marker. However, CF is a multi-system disease and the extent to which simultaneously modeling growth and nutrition markers improves PE predictive accuracy is unknown. Furthermore, it is unclear which routinely collected clinical indicators of growth and nutrition in early life predict PE onset in CF.

Methods: Using a longitudinal cohort of 17,100 patients aged 6-20 years (US Cystic Fibrosis Foundation Patient Registry; 2003-2015), we fit a univariate joint model of lung-function decline and PE onset and contrasted its predictive performance with a class of multivariate joint models that included combinations of growth markers as additional submodels. Outcomes were longitudinal lung function (forced expiratory volume in $1 \mathrm{~s}$ of \% predicted), percentiles of body mass index, weight-for-age and height-for-age and PE onset. Relevant demographic/clinical covariates were included in submodels. We implemented a univariate joint model of lung function and time-to-PE and four multivariate joint models including growth outcomes.

Results: All five joint models showed that declining lung function corresponded to slightly increased risk of PE onset (hazard ratio from univariate joint model: $0.97, P<0.0001$ ), and all had reasonable predictive accuracy (crossvalidated area under the receiver-operator characteristic curve $>0.70$ ). None of the growth markers alongside lung function as outcomes in multivariate joint modeling appeared to have an association with hazard of PE. Jointly modeling only lung function and PE onset yielded the most accurate (area under the receiver-operator characteristic curve $=0.75$ ) and precise (narrowest interquartile range) predictions. Dynamic predictions were accurate across forecast horizons (0.5, 1 and 2 years) and precision improved with age.

(Continued on next page)
\end{abstract}

\footnotetext{
* Correspondence: Rhonda.Szczesniak@cchmc.org

${ }^{3}$ Division of Pulmonary Medicine, Cincinnati Children's Hospital Medical

Center, Cincinnati, OH, USA

${ }^{4}$ Department of Pediatrics, University of Cincinnati, Cincinnati, $\mathrm{OH}$, USA

Full list of author information is available at the end of the article
}

(c) The Author(s). 2020 Open Access This article is licensed under a Creative Commons Attribution 4.0 International License, which permits use, sharing, adaptation, distribution and reproduction in any medium or format, as long as you give appropriate credit to the original author(s) and the source, provide a link to the Creative Commons licence, and indicate if changes were made. The images or other third party material in this article are included in the article's Creative Commons licence, unless indicated otherwise in a credit line to the material. If material is not included in the article's Creative Commons licence and your intended use is not permitted by statutory regulation or exceeds the permitted use, you will need to obtain permission directly from the copyright holder. To view a copy of this licence, visit http://creativecommons.org/licenses/by/4.0/ The Creative Commons Public Domain Dedication waiver (http://creativecommons.org/publicdomain/zero/1.0/) applies to the data made available in this article, unless otherwise stated in a credit line to the data. 
(Continued from previous page)

Conclusions: Including growth markers via multivariate joint models did not yield gains in prediction performance, compared to a univariate joint model with lung function. Individualized dynamic predictions from joint modeling could enhance physician monitoring of CF disease progression by providing PE risk assessment over a patient's clinical course.

Keywords: Dynamic prediction, Functional data analysis, Medical monitoring, Multivariate longitudinal data, Registry analysis, Time-to-event

\section{Background}

Cystic fibrosis (CF) is a chronic lung disease in which death commonly results from respiratory failure [1]. A cycle of prolonged and acute drops in lung function marks the clinical course of CF [2], most notably during adolescence and early adulthood [3-5]. Forced expiratory volume in $1 \mathrm{~s}$ of $\%$ predicted (hereafter, $\mathrm{FEV}_{1}$ ), is the primary surrogate of lung function and strongest predictor of mortality in the CF population [6]. $\mathrm{FEV}_{1}$ remains an important outcome in clinical trials and the most relevant clinical indicator in monitoring lung function decline [7]. Fitting longitudinal $\mathrm{FEV}_{1}$ trajectories is critical to understanding disease progression, but it can be difficult to accurately depict the substantial variation that this marker exhibits both between patients and within an individual patient over time (Fig. 1). It is also of clinical importance to identify an acute respiratory event referred to as a pulmonary exacerbation (PE), which frequently corresponds to sharp decreases in lung function. Diagnosis of a PE depends on various factors corresponding to lung function and nutritional status, often resulting in a patient being hospitalized and administered intravenous antibiotics [8]. Further, patients who experience a PE often fail to recover to their corresponding pre-event (i.e., "baseline") levels of $\mathrm{FEV}_{1}$ [9]. For these reasons, it has been desirable in epidemiologic studies to identify risk factors for having a PE.

Although maintaining lung function and minimizing risk of PEs are essential to survival, CF is a multi-system disease in which malnutrition and poor growth have been shown to adversely impact pulmonary function [10]. Body mass index percentile (BMIp) is the primary nutritional indicator in $\mathrm{CF}$ [11]; guidelines published by the CF Foundation recommend maintaining BMIp >50th percentile [12]. A recent $C F$ registry analysis found that weight-for-age (WFA) and height-for-age (HFA) percentiles more accurately identify CF patients of poor nutritional status and stunting, respectively, compared to BMI percentiles [13].

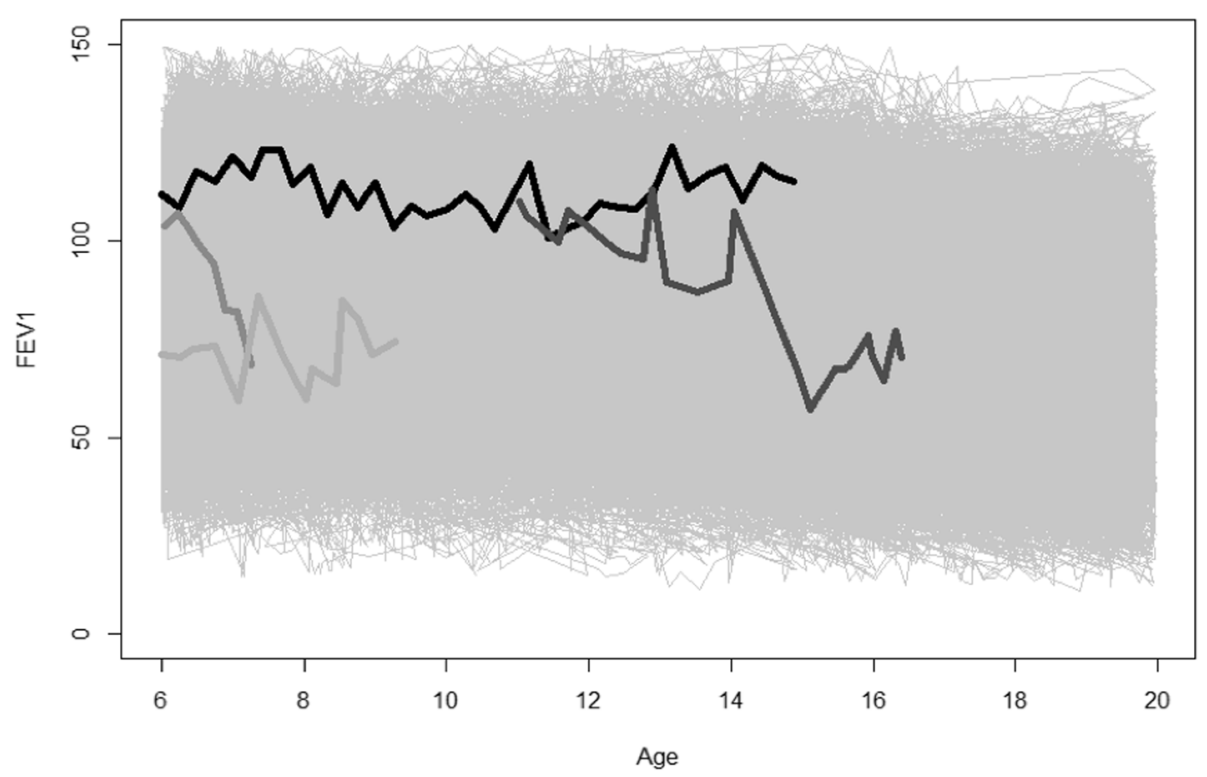

Fig. 1 Lung function profiles over time. FEV1 (\% predicted on the y-axis) is shown against time (age, in years, on the x-axis). Four representative patients are shown with thicker and darker-shaded trajectories, while remaining patients' trajectories are thinner and in light gray. Two of the selected profiles (which appear lower overall, compared to the other two profiles) correspond to two individuals who experienced pulmonary exacerbations 
These and other studies have collectively demonstrated that multiple causes of disease progression are typically observed from indicators of pulmonary and nutritional decline, yet it remains unclear how the underlying longitudinal processes collectively evolve over time. Joint modeling is a popular statistical approach used to link longitudinal and time-to-event processes and is usually implemented through shared parameters [14]. These models assume that the longitudinal and event processes are correlated through random effects [15]. In particular, we describe the evolution over time for the longitudinal outcomes with a mixed-effects model. Then, we use these estimated evolutions as time-varying covariates in the survival model.

Novel applications of so-called univariate joint models (refers to a single longitudinal marker being modeled simultaneously with an event outcome) in CF epidemiologic studies have yielded new insights and predictions regarding how changes in $\mathrm{FEV}_{1}$ are associated with survival (i.e., time to death/lung transplant) [16-18]. Although these applications elucidate CF disease progression into its end stage, little is known about the utility of joint models in assessing onset of PEs; these events initialize earlier in life but could be attributable to downstream impacts. Furthermore, to our knowledge, the association of one or more longitudinal outcomes with onset of PE has not yet been addressed. Univariate joint modeling potentially ignores the fact that different markers of interest, specifically those related to growth and nutrition status, could also have an influence on PE in combination.

Due to computational advancements, such as new software packages [19], joint models have enjoyed a renaissance in the last decade [20]. In this paper, we focus on multiple longitudinal outcomes and their potential to predict PE onset in children with CF. Specifically, we propose a multivariate joint model of $\mathrm{FEV}_{1}$ and markers of nutritional status with time-to-PE. The objective of our study was to construct these models to estimate how commonly collected markers of these longitudinal processes evolve through the clinical course of CF, and to compare results from these models regarding predictive accuracy. We utilize data from the CFF patient registry and provide implementation of the joint models as supplemental material.

\section{Methods}

\section{Cohort description}

The CF cohort for this empirical study consists of patients with longitudinal data recorded from childhood until early adulthood (ages: 6-20 years) in the US Cystic Fibrosis Foundation Patient Registry (CFFPR) between January 1, 2003, and December 31, 2015. Patients younger than 6 years of age were excluded due to potentially unreliable pulmonary function testing. To focus the study on the most modern era of CF care, we considered available data from 2003 onward. A detailed description of this registry and its contents is provided elsewhere [21].

\section{Outcomes}

To build the multivariate joint model for this cohort, we focused on established outcomes of lung function and nutrition. Outcomes on nutrition and growth, available from the CFFPR and computed based on formulas from the Centers for Disease Control and Prevention, included the aforementioned percentiles for BMIp, WFA and HFA. Observed $\mathrm{FEV}_{1}$ was expressed as percentage of predicted using established reference equations for age, sex and race [22, 23]. Occurrence of first PE, the event outcome, was subject to censoring and considered to have occurred if documented in the CFFPR as warranting treatment with intravenous antibiotics in the hospital.

\section{Covariates}

Covariates from the aforementioned literature were considered, as well as findings from analyses specified in the most recent CFFPR report. These included genotype (F508del homozygous, heterozygous or neither/unknown), sex, Hispanic ethnicity, socioeconomic status (SES, having only state/federal or no insurance was recorded as 1 , and 0 otherwise); time-varying covariates included use of pancreatic enzymes (corresponding to pancreatic insufficiency), CF-related diabetes (CFRD, with or without fasting hyperglycemia), positive cultures for methicillin-resistant Staphylococcus aureus (MRSA) and Pseudomonas aeruginosa $(\mathrm{Pa})$.

Baseline was defined as the time at which all longitudinal outcomes were first recorded during the eligibility period. A birth cohort variable was used to account for potential delayed entry into the registry and changes due to advancements in CF care and therapeutics approvals. Another time-varying covariate was used to account for irregular sampling bias, potentially induced by patients having a varying number of clinical encounters over time; for a given patient and encounter, this variable was the total number of encounters within the prior year. Data acquired after lung transplant were excluded.

\section{Descriptive analysis}

Descriptive statistics, including median (Q1-Q3) for continuous variables and $\mathrm{n}(\%)$ for categorical variables, were used to summarize cohort characteristics and extent of follow-up available for each patient. 


\section{Joint model setup and notation}

Prior to multivariate joint modeling, we performed a univariate joint model that included only $\mathrm{FEV}_{1}$ and time-to-first PE. To model the association between combinations of longitudinal $\mathrm{FEV}_{1}$ and other outcomes together with time-to-first $\mathrm{PE}$, we examined multivariate joint models. We describe the notation using a particular multivariate joint model with two longitudinal outcomes, which can be generalized to the case of three outcomes (e.g. $\mathrm{FEV}_{1}$, WFA and HFA). We assume $y_{1 i}(t)$ and $y_{2 i}(t)$ to be the follow-up measurements (e.g., $\mathrm{FEV}_{1}$ and BMIp, respectively) for patient $i(i=1, \ldots, n)$ at a specific time point $t$. We follow the framework of mixed-effects models to model the longitudinal outcomes:

$$
y_{1 i}(\mathrm{t})=x_{1 i}^{T}(t) \beta_{1}+z_{1 i}^{T}(t) b_{1 i}+\varepsilon_{1 i}(t)=m_{1 i}(t)+\varepsilon_{1 i}(t)
$$

and

$$
y_{2 i}(\mathrm{t})=x_{2 i}^{T}(t) \beta_{2}+z_{2 i}^{T}(t) b_{2 i}+\varepsilon_{2 i}(t)=m_{2 i}(t)+\varepsilon_{2 i}(t),
$$

where $x_{1 i}^{T}(t)$ and $x_{2 i}^{T}(t)$ denote the design vectors for the fixed effects regression coefficients $\beta_{1}$ and $\beta_{2}$. Moreover, $z_{1 i}^{T}(t)$ and $z_{2 i}^{T}(t)$ denote the design vectors for the random effects $b_{2 i}$ and $b_{1 i}$. We included natural cubic splines to accommodate nonlinear progression of different covariate levels over age with respect to each longitudinal outcome, using an approach similar to our previous work in modeling nonlinear age-related $\mathrm{FEV}_{1}$ [5]) [24]). Furthermore, the longitudinal outcomes FEV $_{1}$ and BMIp are correlated through the random effects $b_{i}=$ $\left\{b_{1}, b_{2 i}\right\}$, where a multivariate normal distribution is assumed.

For the PE part, we assume $T_{i}$ to be the observed failure time for patient $i$, as $T_{i}=\min \left(T_{i}^{*}, C_{i}\right)$ where $T_{i}^{*}$ indicates the true failure time of individual $i^{\text {th }}$ experiencing the event (occurrence of $\mathrm{PE}$ ) and $C_{i}$ the censored time. We assume a proportional hazard model for the risk of the event:

$$
\mathrm{h}_{\mathrm{i}}(\mathrm{t})=\mathrm{h}_{0}(\mathrm{t}) \exp \left\{\omega_{\mathrm{i}}^{\mathrm{T}} \gamma+\mathrm{a}_{1} \mathrm{~m}_{1 \mathrm{i}}(\mathrm{t})+\mathrm{a}_{2} \mathrm{~m}_{2 \mathrm{i}}(\mathrm{t})\right\}
$$

where $\omega_{i}^{T}$ denotes row vectors of the design matrix of the baseline covariates,$\gamma$ is the corresponding regression coefficients vector, and $a_{1}$ and $a_{2}$ are the coefficients that connect the longitudinal and event processes. In our case, these parameters represent the strength of the association between $\mathrm{FEV}_{1}$ and BMIp with onset of PE. To better understand the connection between the event and longitudinal parts in our model, we explain the meaning of the $a_{1}$ and $a_{2}$ parameters. Specifically, for a one unit increase in the underlying value of $\mathrm{FEV}_{1}$ for patient $i$, the hazard ratio is $\exp \left(\mathrm{a}_{1}\right)$ assuming that the baseline covariates and BMIp remain the same. Similarly, $\exp \left(\mathrm{a}_{2}\right)$ is the hazard ratio when the underlying value of BMIp for the $i$-th patient is increased by one unit assuming that the baseline covariates and $\mathrm{FEV}_{1}$ remain the same.

\section{Implementation}

We employed Markov-Chain Monte-Carlo (MCMC) via Gibbs sampling using the 'JMbayes' package (Version 0.8-82) in R (Version 3.5.3., R Foundation for Statistical Computing, Vienna Austria) to obtain the parameters from the respective posterior distributions under the multivariate joint model [19]. The highest posterior density (HPD) and accompanying standard errors were used to estimate each parameter of interest. Due to the large patient sample size that led to memory problems, we randomly divided the dataset into three parts (each part had similar percentage of PE at onset). Each of the datasets was fitted separately, and for the final results we combined the MCMC samples as described previously [25]. Code implementation is provided as supplemental material. In total, we fit five joint models wherein PE onset was the event: i) only $\mathrm{FEV}_{1}$; others included ii) BMIp; iii) HFA; iv) WFA; v) WFA and HFA. The multivariate joint models in (ii) - (iv) were implemented to evaluate the impact of different measures of nutritional status on predicting PE onset. Due to the large number of parameters in scenario (v), we assumed a less flexible evolution for $\mathrm{FEV}_{1}$ and BMIp over time. In particular, we postulate natural cubic splines with two degrees of freedom to estimate this evolution instead of natural cubic splines with three degrees of freedom assumed in the other scenarios.

\section{Evaluating predictive performance}

We calculated the area under the receiver-operator characteristic curve (AUC) to evaluate predictive accuracy of each model with respect to $\mathrm{PE}$ events using ten-fold cross validation. A large value of AUC indicates the preferred model. To obtain correct estimates of the AUC we performed a 5-fold cross validation procedure, wherein each time we selected 700 individuals from the large data set and we split it in five subsets. Each time we fitted the model in four of the subsets, we calculated the AUC in the subset that was excluded. The calculation of the AUC was performed at 12 and 16 years of age with prediction windows of $0.5,1,2$ years, in order to mimic clinically meaningful age strata and prediction windows. This procedure was repeated 100 times. Appropriate diagnostics for joint models were examined. The code of the analysis and cross validation are shown in the Supplement.

\section{Results}

\section{Analysis cohort}

There were 16,455 patients contributing 245,513 observations to the analysis cohort (Table 1). Patients who 
Table 1 Cohort characteristics according to PE onset*

\begin{tabular}{|c|c|c|c|}
\hline Characteristic at Baseline & Had PE during follow up $(n=5510)$ & PE-free during follow up $(n=10,945)$ & Overall $(n=16,455)$ \\
\hline \multicolumn{4}{|l|}{ Genotype^ } \\
\hline Homozygous & $53.0 \%$ & $45.6 \%$ & $48.1 \%$ \\
\hline Heterozygous & $36.4 \%$ & $39.1 \%$ & $38.2 \%$ \\
\hline None/unknown & $10.6 \%$ & $15.3 \%$ & $13.7 \%$ \\
\hline Male sex^ & $47.4 \%$ & $53.7 \%$ & $51.6 \%$ \\
\hline Hispanic & $9.2 \%$ & $8.5 \%$ & $8.7 \%$ \\
\hline \multicolumn{4}{|l|}{ Birth cohort^ } \\
\hline$<1981$ & $16.7 \%$ & $19.8 \%$ & $18.8 \%$ \\
\hline 1981-1989 & $31.6 \%$ & $22.2 \%$ & $25.3 \%$ \\
\hline 1990-1994 & $22.0 \%$ & $13.9 \%$ & $16.6 \%$ \\
\hline 1995-1999 & $24.6 \%$ & $26.9 \%$ & $26.1 \%$ \\
\hline$>1999$ & $5.1 \%$ & $17.2 \%$ & $13.2 \%$ \\
\hline Age at entry, years $\wedge$ & $8.7(6.2-13.0)$ & $7.9(6.2-14.0)$ & $8.2(6.2-13.6)$ \\
\hline LOW SES & $54.0 \%$ & $52.4 \%$ & $49.8 \%$ \\
\hline MRSA^ & $9.5 \%$ & $6.4 \%$ & $7.4 \%$ \\
\hline $\mathrm{Pa} \wedge$ & $24.2 \%$ & $17.3 \%$ & $19.6 \%$ \\
\hline $\mathrm{CFRD} \wedge$ & $5.1 \%$ & $3.2 \%$ & $3.9 \%$ \\
\hline Pancreatic insufficient^ $\wedge$ & $32.3 \%$ & $42.3 \%$ & $39.0 \%$ \\
\hline $\mathrm{FEV}_{1}, \%$ predicted $\wedge$ & $83.8(67.3-98.0)$ & $93.5(79.3-105.2)$ & $90.6(74.9-103.2)$ \\
\hline BMIp, percentile^ & $43.1(19.9-67.0)$ & $51.1(27.3-73.4)$ & $48.6(24.8-71.3)$ \\
\hline WFA, percentile^ & $29.1(10.2-55.0)$ & $38.9(16.7-64.7)$ & $35.5(14.3-61.7)$ \\
\hline HFA, percentile^ & $24.5(8.4-49.8)$ & $32.4(12.3-59.5)$ & $29.7(10.7-56.7)$ \\
\hline Length of follow up, years $\wedge$ & $1.9(0.5-4.4)$ & $3.1(1.0-6.3)$ & $2.6(0.8-5.7)$ \\
\hline Alive^^ & $99.9 \%$ & $99.7 \%$ & $99.8 \%$ \\
\hline
\end{tabular}

Abbreviations: BMIp body mass index percentile, CFRD cystic fibrosis related diabetes, FEV ${ }_{1}$ forced expiratory volume in $1 \mathrm{~s}, H F A$ height for age, MRSA methicillinresistant Staphylococcus aureus, Pa Pseudomonas aeruginosa, PE pulmonary exacerbation, SES socioeconomic status, WFA weight for age. * Values at first recorded entry in the database are used for all patients except for follow-up and death; results for continuous and categorical variables are expressed as median (Q1-Q3) and \% over column total, respectively. Characteristics marked as $\wedge$ imply $P<0.05$ for comparison between PE and PE-free groups. Between-group frequencies compared using Chi-square test of independence; continuous variables compared between groups using Welch two-sample t-test

had a PE during follow-up tended to be female and had the F508del homozygous genotype. They had slightly higher proportions of infections with MRSA and Pa than patients who did not have a PE during follow up. By contrast, patients who were PE-free during follow-up had a higher reported use of pancreatic enzymes and had higher levels of lung function and better nutrition status at baseline. Extent of follow-up also differed according to PE onset. Representative trends of lung function decline according to PE status are shown in Fig. 1 and reflect summary results from Table 1. Median (95\% CI) age of PE onset was 19.5 (19.3-19.6) years (Fig. 2).

\section{Joint model estimates}

Each Gibbs sampler ran for 900 iterations, and the first 600 iterations were discarded for burn-in. Shared parameter estimates, which represent associations between each longitudinal process and the PE event, are shown for all five fitted joint models (Table 2). The log-hazard estimates of the shared parameters, which were introduced in Section 3.2, indicate that $\mathrm{FEV}_{1}$ was consistently and negatively associated with PE onset. Corresponding hazard ratio estimates ranged from 0.96 to 0.97 , suggesting a small association between changes in the $\mathrm{FEV}_{1}$ trajectory and PE onset. Jointly modeling BMIp, WFA or HFA as shown in respective models (ii) - (iv) did not impact association between $\mathrm{FEV}_{1}$ and PE onset, and their associations with $\mathrm{PE}$ onset did not reach statistical significance.

The univariate joint model (i) in Table S1 indicated that being Hispanic and having lower SES, MRSA and CFRD and using pancreatic enzymes corresponded to worse overall $\mathrm{FEV}_{1}$. There were nonlinear associations, as reflected by spline coefficients, between each of genotype, sex, SES, MRSA, Pa and CFRD and $\mathrm{FEV}_{1}$ decline. In the PE event submodel, patients who were male, used pancreatic enzymes, had lower SES, infection with MRSA and $\mathrm{Pa}$ and were diagnosed with CFRD had 


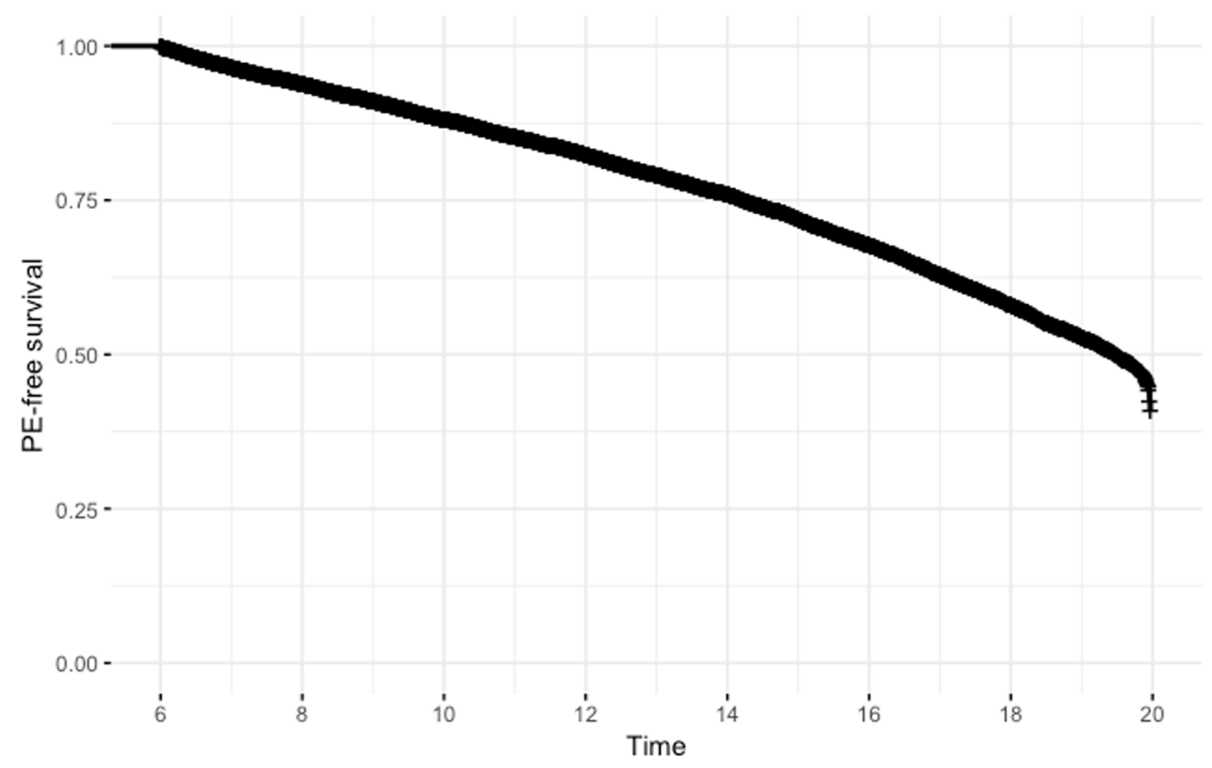

Number at risk

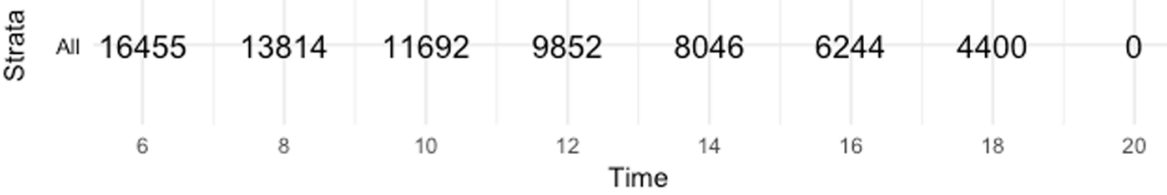

Fig. 2 Kaplan-Meier plot of time to first recorded pulmonary exacerbation. Time is expressed as age (in years on the x-axis) and PE-free survival probability (y-axis) corresponds to probability of not experiencing a pulmonary exacerbation. Data shown include all patients

greater risk of PE onset. The multivariate joint models, each of which included a longitudinal submodel for $\mathrm{FEV}_{1}$, had similar association results for the clinical/demographic covariates and shared parameters regarding $\mathrm{FEV}_{1}$.

The multivariate joint model (ii) in Table S2, which included a longitudinal submodel for BMIp, showed that being born into an older birth cohort, taking pancreatic enzymes and having more frequent clinic visits corresponded to higher BMIp. There were nonlinear associations between each of genotype, SES, birth cohort and $\mathrm{Pa}$ with BMIp trajectory. In Table S3, using height in multivariate joint model (iii), we found that having a F508del homozygous genotype and belonging to an older birth cohort were positively associated with overall HFA. By contrast to the BMIp submodel, having more frequent clinic visits corresponded to lower HFA. Similar nonlinear associations were observed in the BMIp and HFA submodels. Results for the WFA submodel in (iv) were also similar (Table S4). The multivariate joint model in (v), compared to models (iii) and (iv), had slight changes in associations shown in the WFA submodel; however, the HFA submodels were similar.

Table 2 Associations between longitudinal processes and risk of PE*

\begin{tabular}{|c|c|c|c|c|}
\hline \multirow[b]{2}{*}{ Joint Model } & \multicolumn{4}{|c|}{$\log (\mathrm{HR})$ estimates (SE) and $P$-value } \\
\hline & $\overline{\mathrm{FEV}_{1}}$ & BMlp & WFA & HFA \\
\hline I- Null & $-0.0305(5 \mathrm{e}-04) P<0.001$ & - & - & - \\
\hline II - BMlp & $-0.0324(6-04) P=0.002$ & $-0.0004(3-04) P=0.988$ & - & - \\
\hline III - WFA & $-0.0334(7 \mathrm{e}-04) P=0.003$ & - & $0.0006(2 \mathrm{e}-04) P=0.761$ & - \\
\hline IV - HFA & $-0.0308(5 \mathrm{e}-04) P=0.001$ & - & - & $0.0004(1 \mathrm{e}-04) P=0.927$ \\
\hline V - WFA and HFA & $-0.0327(6 \mathrm{e}-04) P=0.005$ & - & $0.0022(9 \mathrm{e}-04) P=0.683$ & $-0.0019(1 \mathrm{e}-03) P=0.737$ \\
\hline
\end{tabular}

Abbreviations: $B M I p$ body mass index percentile, $F E V$, forced expiratory volume in $1 \mathrm{~s}, H F A$ height-for-age percentile, $H R$ hazard ratio, $P E$ pulmonary exacerbation, WFA weight-for-age percentile. *Associations estimated as log hazard using the posterior mean (SE) with corresponding $p$-value in the Bayesian sense. Log-hazard estimates $>0(<0)$ imply positive (negative) association between the longitudinal process and PE onset. Results are reported as HRs in text. Each model includes all covariates (see Methods). All joint model parameter estimates are provided in Supplement S1 


\section{Predictive performance}

The results of the predictive performance of the joint models presented in Table 2 are summarized in Fig. 3. In particular, the AUC of each model is presented assuming a different prediction interval $(0.5,1$ and 2 years), which starts at adolescence or early adulthood (ages 12 and 16 years, respectively). The median AUC values ranged from 0.7 to 0.8 . Moreover, the univariate joint model, assuming only $\mathrm{FEV}_{1}$, provided the highest AUC value and was most robust across age strata and prediction windows. Precision of the predictions was assessed by examining the interquartile ranges of the boxplots in Fig. 3. Precision was lower for predictions done at adolescence, regardless of the interval, compared to precision estimated during young adulthood. Precision was highest under the univariate joint model for predicting PE risk at age 16 years out to 2 years. In terms of overall precision, the multivariate joint models performed similarly well.

Dynamic predictions for an individual CF F508del homozygous patient (born 1990-1994) are shown in Fig. 4 for the multivariate joint model of longitudinal $\mathrm{FEV}_{1}$ and BMIp. Her first recorded $\mathrm{FEV}_{1}$ and BMIP were $98.5 \%$ predicted and at the 48.2 percentile, respectively. She began taking pancreatic enzymes and had a positive culture for $\mathrm{Pa}$ around 10 years old. Her monitored outcomes are depicted across four clinic visits from ages 8.2 to 14.3 years, along with probability of not having a PE over follow up. Her long-term risk of PE appears higher during an earlier visit (e.g., PE-free probability is 0.6), compared to later visits that are informed by dynamic predictions from the multivariate joint models (e.g., PE-free probability increased to 0.8). Commensurate with these results are the changes in her $\mathrm{FEV}_{1}$ and BMIp trajectories, which imply minimal rate of decline and improving nutritional status, respectively. She did not experience a PE event during follow up. Her projected PE-free probabilities over follow up using a multivariate joint model replacing BMIp with WFA and HFA were similar (Fig. 5); however, precision with which PE-free probability could be estimated was decreased using this combination of markers rather than BMIp.

\section{Discussion}

Past epidemiologic studies of PE onset have largely focused on explanation of risk factors rather than identifying predictors of this event. Clinical consensus on PE symptoms is lacking. Acute drops in lung function and changes in nutritional status have been recognized as key determinants [26]; however, relative contributions of these markers to prediction of PE onset have not been studied. Further, markers of growth/nutritional status (WFA, HFA and BMIp) and lung function $\left(\mathrm{FEV}_{1}\right)$ are endogenous variables. In this epidemiologic study, we assessed predictive performance of these markers using a series of joint models. Our novel application confirmed the reliance of changes in the $\mathrm{FEV}_{1}$ trajectory on predicting PE events but revealed the clinical utility that these novel models could have for monitoring multiple indicators of disease progression for an individual patient. In parallel, our application highlights that, although pancreatic enzyme use is commonly employed as

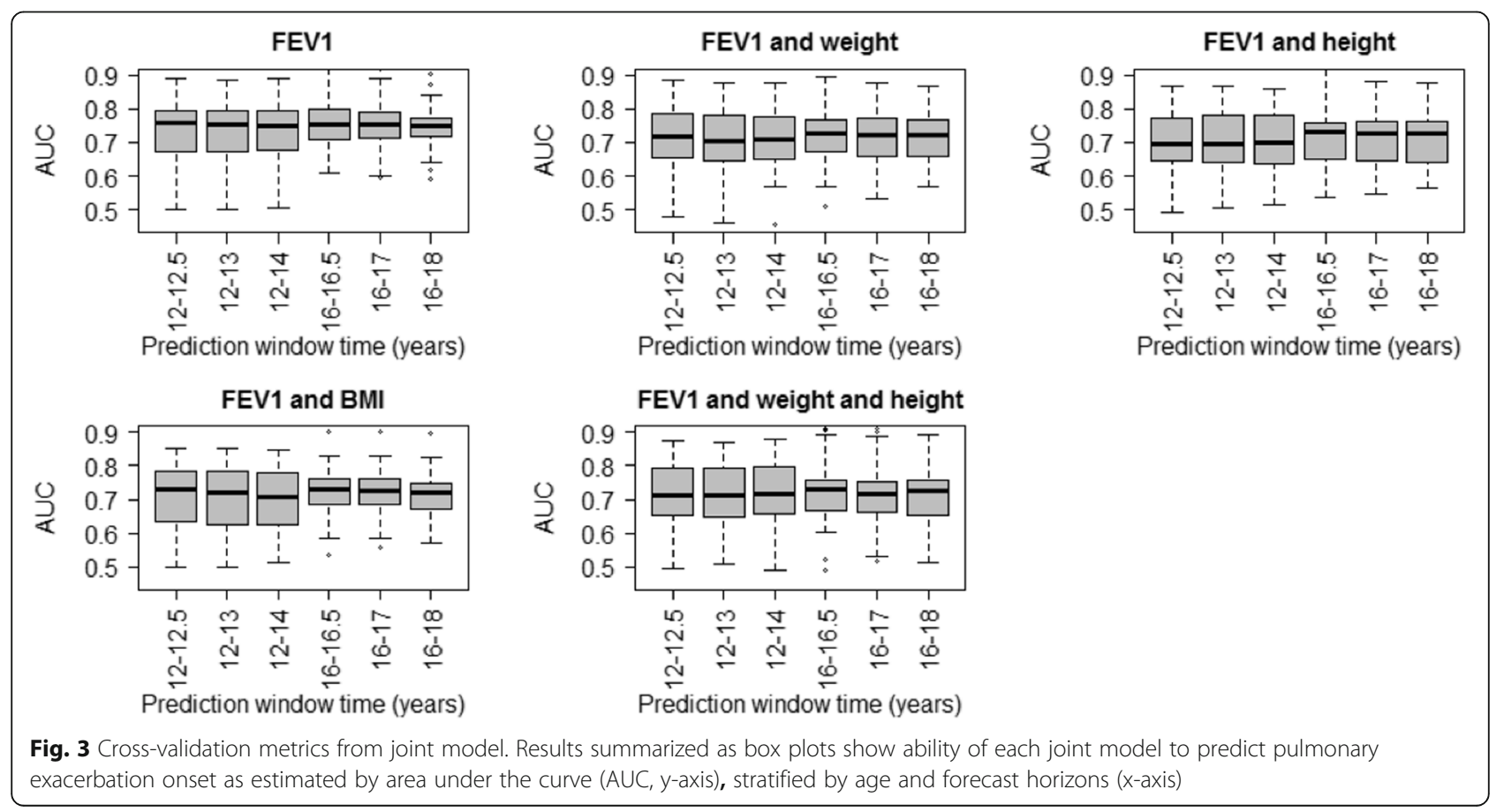




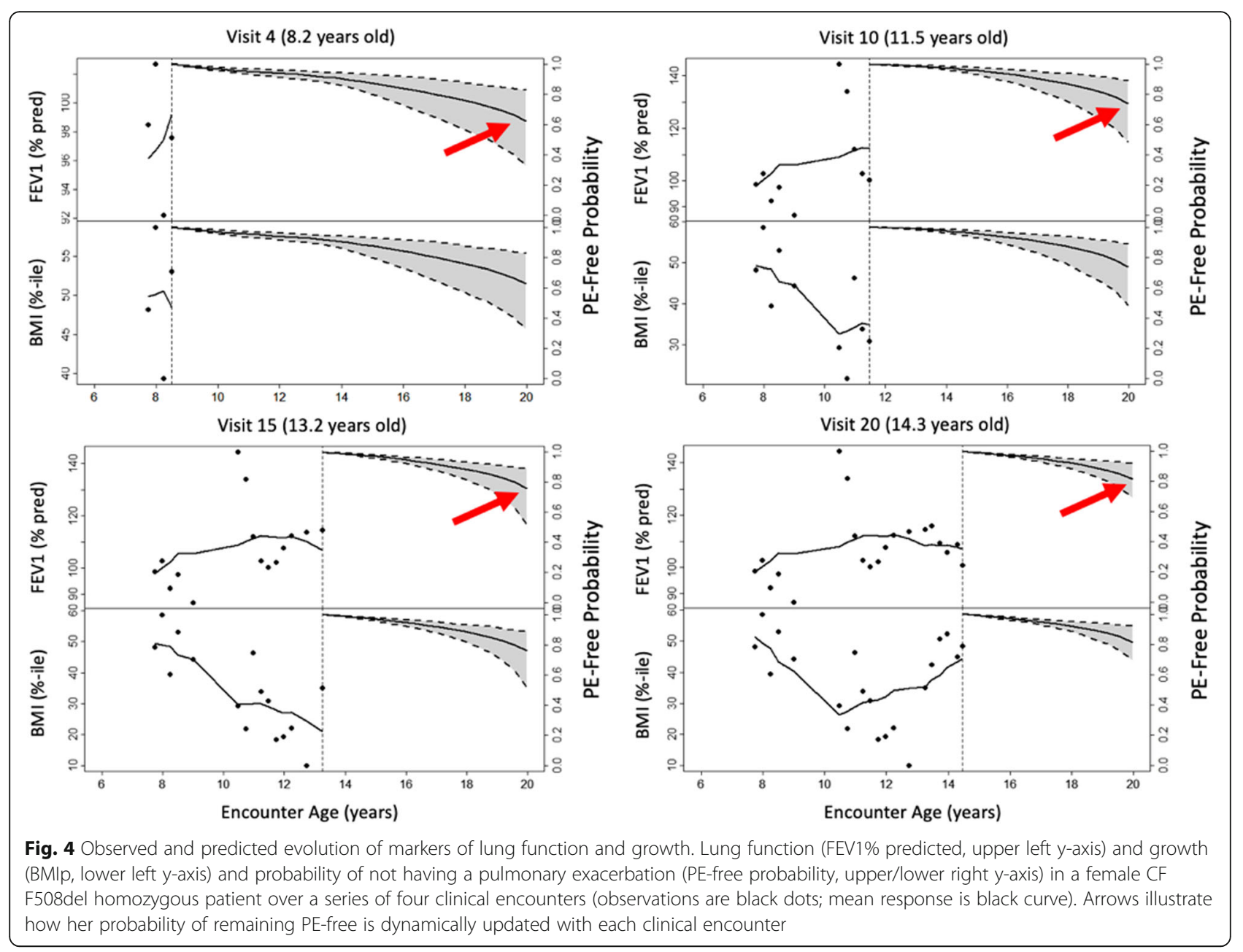

a marker of pancreatic insufficiency, their use was associated with lower PE frequency over follow-up (Table 1). It is possible that pancreatic enzyme use is also a marker of receiving higher-quality healthcare.

We found that the univariate joint model, which utilized $\mathrm{FEV}_{1}$ and $\mathrm{PE}$ event submodels without accounting for growth/nutritional status, produced the most accurate and precise predictions of all models considered. In addition, these predictions were largely robust across a range of age and prediction window strata. A potential reason for this finding is that the diagnosis of PE events may rely more heavily upon acute changes in lung function than changes in nutritional status. Changes in growth are relatively slow over time, compared to precipitous drops in lung function, which could be responsible for the lack of improved predictive performance when adding WFA, HFA or BMIp. Of the three markers available from the CFFPR to study growth/nutritional status, it is plausible that BMIp would be most likely to improve prediction of $\mathrm{PE}$ onset (after including $\mathrm{FEV}_{1}$ ); however, it appears that including BMIp as a submodel introduces additional noise into the joint modeling of these processes, thereby making predictions less accurate and more variable (Fig. 3). In addition, associations between clinical/demographic covariates and each outcome were largely consistent across $\mathrm{FEV}_{1}$ submodels. This finding further suggests that WFA, HFA and BMIp do not play a large role in clinical diagnosis of PE; however, the findings from the study aimed at prediction cannot serve as a substitute for assessing the causal effects of nutrition [27]. The results do warrant further investigation of a causal model examining the extent to which nutrition and growth mediate the relationship between lung function and PE onset. Understanding these causal underpinnings are a separate but important area of future work.

The multivariate joint models afforded the opportunity to obtain dynamic predictions at the individual patient level (Fig. 4); however, using multiple nutrition markers may decrease precision of PE predictions (Fig. 5). The decrease in precision may be due to relatively large variability in each outcome both between patients and within individual patients over time. Both Figs. 4 and 5 illustrate the substantial intra-individual variability in 


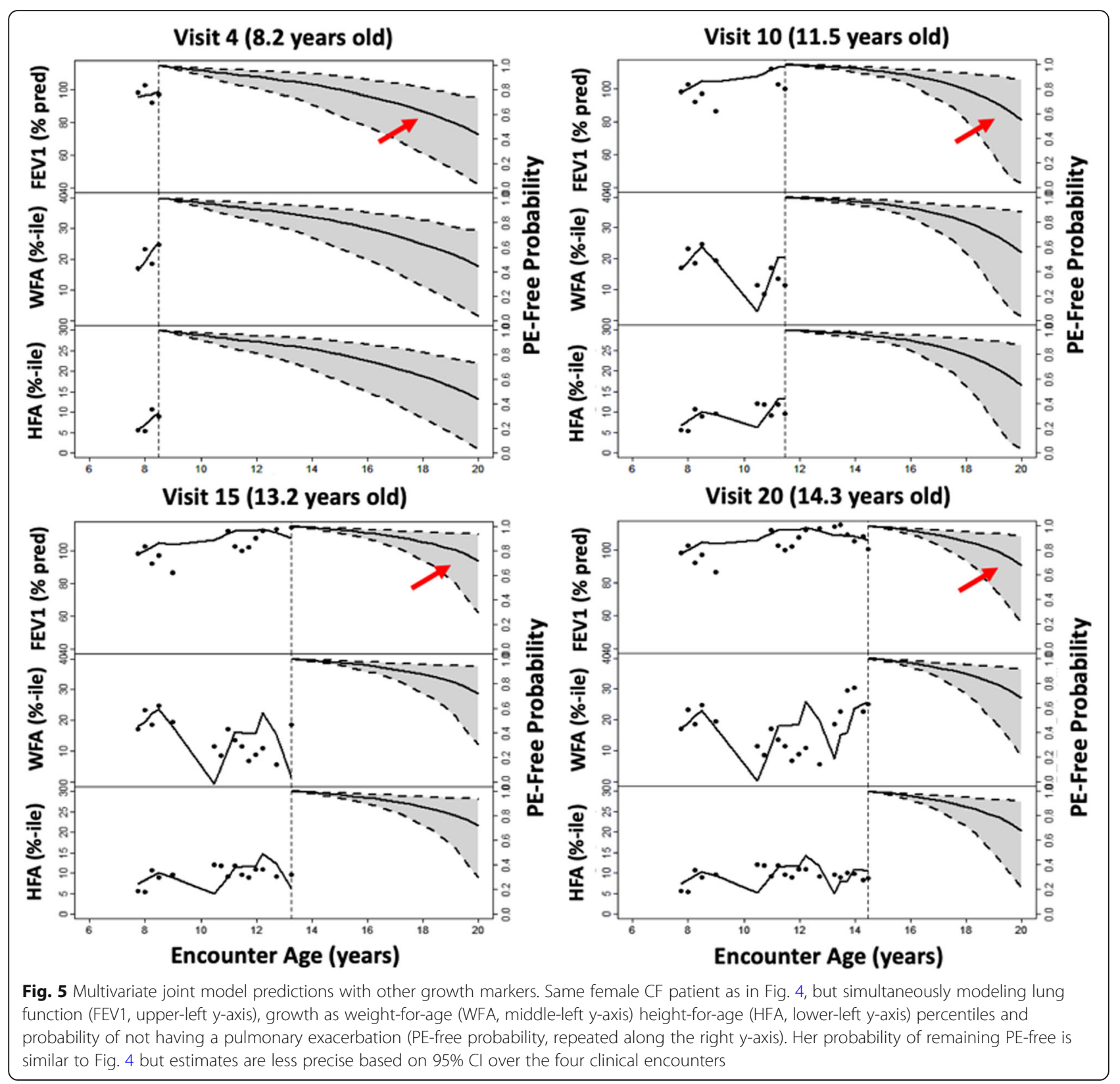

each of the lung function and growth markers. In contrast with joint modeling results, we found that patients who experienced a PE tended to have lower WFA, HFA

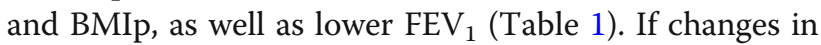
nutrition markers are less influential in making a PE diagnosis than change in lung function, the contrasting results could reflect differences between association and prediction [27]. All of the outcome markers could be associated with PE onset but not all markers may be necessary to accurately predict it. The focus of the manuscript was to investigate the predictive performance of the different joint models. Hence, we did not assess the fit of those models. Using these dynamic predictions revealed the heterogeneous nature of CF disease progression but also highlighted the benefit of updated predictions across clinic visits. The ability to simultaneously monitor multiple markers of disease progression, which may serve as a clinical decision support tool, provides an advantage of the multivariate joint model over the univariate joint model and the more conventional Cox model. An area of future study could be the additional consideration of other markers of lung function as predictors, including forced vital capacity (FVC), the $\mathrm{FEV}_{1} / \mathrm{FVC}$ ratio and forced expiratory flow at 25-75\% (referred to as FEF25-75). Furthermore, in this research we assumed the underlying value of the 
biomarkers (FEV 1 , WFA, HFA and BMIp) to be associated with PE. A special feature of these biomarkers is that they are time-dependent, therefore the assumption of the underlying value might be too strict. Future research could focus on investigating whether other structures, such as the slope of these markers, could provide a stronger association with $\mathrm{PE}$ and a better predictive performance.

A limitation of this study is that the models cannot be used to draw conclusions about recurrent PE events, since the PE event submodel estimates time-to-first PE. Although this focus simplifies analyses, time-to-first PE during follow up tended to occur in early adulthood (Fig. 2). It is possible that a portion of patients experienced a PE prior to follow-up available in the data (e.g., patients born in older birth cohorts). In addition, the advent of newer therapies and/or quality improvement initiatives likely increase the overall trajectories of outcomes studied. We accounted for these potential sources of bias by including levels of birth cohort through linear and nonlinear terms in each of the longitudinal submodels (Tables S1-S5). Consequently, including higher-order associations through splines required additional degrees of freedom. Future work is needed on covariate selection strategies in the context of multivariate joint models $[28,29]$. Fitting these more complex models to a large number of patients followed over long periods of time creates a computational challenge. We addressed this issue by partitioning into distinct sets of patients, which was the only feasible manner to estimate the model parameters; however, the bootstrap approach that we employed does not account for all the variability of the outcomes. Lastly, the multivariate joint models presented here allow for different patterns of missing data among the longitudinal outcomes but assume that the missingness is conditional on observed data only (i.e., missing at random), specifically occurrence of PE. It is possible that missingness could be related to patient severity. A recent study demonstrates that linear mixed effects modeling, which is the framework for the longitudinal submodels used in this application, can alleviate this potential source of bias [30].

\section{Conclusion}

The univariate joint modeling of the CFFPR demonstrates the reliance of PE diagnosis on $\mathrm{FEV}_{1}$, and multivariate joint modeling of $\mathrm{FEV}_{1}$ and other outcomes related to nutritional status and growth can be used for routine medical monitoring of an individual patient and dynamic assessment of PE risk over time.

\section{Supplementary information}

Supplementary information accompanies this paper at https://doi.org/10. 1186/s12890-020-1177-z.

Additional file 1: Table S1. Joint Model with Longitudinal FEV1 and PE onset. Table S2. Joint Model with Longitudinal FEV1, BMIp and PE onset.
Table S3. Joint Model with Longitudinal FEV1, HFA and PE onset. Table S4. Joint Model with Longitudinal FEV1, WFA and PE onset. Table S5 Joint Model with Longitudinal FEV1, WFA, HFA and PE onset.

Additional file 2. Multivariate joint modeling to identify markers of growth and lung function decline that predict cystic fibrosis pulmonary exacerbation onset.

\section{Abbreviations}

AUC: Area under the receiver-operator characteristic curve; BMIp: Body mass index percentile; CF: Cystic fibrosis; CFF: CYSTIC Fibrosis Foundation; CFRD: Cystic fibrosis related diabetes; Cl: Confidence interval; FEV $\mathrm{F}_{1}$ : Forced expiratory volume in $1 \mathrm{~s}$ of $\%$ predicted; HFA: Height-for-age percentile; MRSA: Methicillin-resistant Staphylococcus aureus; Pa: Pseudomonas aeruginosa; PE: Pulmonary exacerbation; SES: Socioeconomic status; WFA: Weight-for-age percentile

\section{Acknowledgments}

The authors would like to thank the Cystic Fibrosis Foundation for the use of Cystic Fibrosis Foundation Patient Registry (CFFPR) data to conduct this study. Additionally, we would like to thank the patients, care providers, and clinic coordinators at Cystic Fibrosis centers throughout the United States for their contributions to the CFFPR.

\section{Authors' contributions}

RS, EA and JPC developed the concept of the study. RS and EA performed all statistical analyses described in the paper. JPC provided clinical guidance on candidate covariates and interpretation of results. All authors read and approved the final versions of the manuscript and supplemental files.

\section{Funding}

This study was funded by grants K25 HL125954 and R01 HL141286 from the National Heart, Lung and Blood Institute of the National Institutes of Health and R457-CR11 from the Cystic Fibrosis Foundation Research and Development Program. The design of the study, analysis and interpretation of data do not necessarily represent official views of the NIH or CFF.

\section{Availability of data and materials}

Implementation code for the models is provided as supplemental material (Supplementary File 2). In accordance with the CFFPR Information Use Agreement, the authors are not authorized to make the CF data accessible. Inquiries regarding data access may be directed via email to the Cystic Fibrosis Foundation: datarequests@cff.org.

\section{Ethics approval and consent to participate}

The Institutional Review Board at Cincinnati Children's Hospital Medical Center approved the study. Upon local IRB approval of this study, we submitted a data use proposal to the CFF Patient Registry Committee, which undertakes an established peer-review process of every request. We refined our request according to Committee feedback and, once they gave final approval, we received non-identifiable and non-traceable data through an encrypted data delivery Citrix ShareFile system. All individuals diagnosed with CF and associated disorders who receive care at a center accredited by the CFF who have provided informed consent are considered eligible to have their data collected in the CFFPR. Per CFF requirements to acquire data in the CFFPR, each center obtained approval from their local institutional review board and written informed consent and assent from individuals and respective legal guardians as required. The analysis cohort derived from the CFFPR and utilized in this particular study was therefore retrospective in nature and de-identified prior to receiving data from the CFF.

Consent for publication

Not applicable.

\section{Competing interests}

RS serves as a member of the Cystic Fibrosis Foundation Patient Registry Committee. JPC is an employee of the Cystic Fibrosis Foundation. Authors RS and JPC have no other competing interests to declare. EA has no competing interests to declare. 


\section{Author details}

'Department of Biostatistics, Erasmus MC, Rotterdam, The Netherlands. ${ }^{2}$ Cystic Fibrosis Foundation, Bethesda, MD, USA. ${ }^{3}$ Division of Pulmonary Medicine, Cincinnati Children's Hospital Medical Center, Cincinnati, OH, USA. ${ }^{4}$ Department of Pediatrics, University of Cincinnati, Cincinnati, OH, USA. ${ }^{5}$ Division of Biostatistics \& Epidemiology, Cincinnati Children's Hospital Medical Center, Cincinnati, OH, USA.

Received: 7 November 2019 Accepted: 30 April 2020

Published online: 19 May 2020

\section{References}

1. Foundation CF. Cystic Fibrosis Foundation patient registry. Bethesda: Cystic Fibrosis Foundation; 2019.

2. Aaron SD, Stephenson AL, Cameron DW, Whitmore GA. A statistical mode to predict one-year risk of death in patients with cystic fibrosis. J Clin Epidemiol. 2015;68(11):1336-45.

3. Liou TG, Elkin EP, Pasta DJ, Jacobs JR, Konstan MW, Morgan WJ, et al. Yearto-year changes in lung function in individuals with cystic fibrosis. J Cyst Fibros. 2010:9(4):250-6.

4. Vandenbranden SL, McMullen A, Schechter MS, Pasta DJ, Michaelis RL, Konstan MW, et al. Lung function decline from adolescence to young adulthood in cystic fibrosis. Pediatr Pulmonol. 2012;47(2):135-43.

5. Szczesniak RD, McPhail GL, Duan LL, Macaluso M, Amin RS, Clancy JP. A semiparametric approach to estimate rapid lung function decline in cystic fibrosis. Ann Epidemiol. 2013:23(12):771-7.

6. Liou TG, Adler FR, Fitzsimmons SC, Cahill BC, Hibbs JR, Marshall BC. Predictive 5-year survivorship model of cystic fibrosis. Am J Epidemiol. 2001; 153(4):345-52

7. Szczesniak R, Heltshe SL, Stanojevic S, Mayer-Hamblett N. Use of FEV1 in cystic fibrosis epidemiologic studies and clinical trials: a statistical perspective for the clinical researcher. J Cyst Fibros. 2017;16:318-26.

8. Flume PA, Mogayzel PJ Jr, Robinson KA, Goss CH, Rosenblatt RL, Kuhn RJ, et al. Cystic fibrosis pulmonary guidelines: treatment of pulmonary exacerbations. Am J Respir Crit Care Med. 2009:180(9):802-8.

9. Sanders DB, Bittner RC, Rosenfeld M, Hoffman LR, Redding GJ, Goss CH. Failure to recover to baseline pulmonary function after cystic fibrosis pulmonary exacerbation. Am J Respir Crit Care Med. 2010;182(5):627-32.

10. Konstan MW, Butler SM, Wohl ME, Stoddard M, Matousek R, Wagener JS, et al. Growth and nutritional indexes in early life predict pulmonary function in cystic fibrosis. J Pediatr. 2003;142(6):624-30.

11. Lai HJ, Shoff SM. Classification of malnutrition in cystic fibrosis: implications for evaluating and benchmarking clinical practice performance. Am J Clin Nutr. 2008;88(1):161-6

12. Stallings VA, Stark $L$, Robinson KA, Feranchak AP, Quinton H. Clinical practice guidelines on $\mathrm{G}$, et al. evidence-based practice recommendations for nutrition-related management of children and adults with cystic fibrosis and pancreatic insufficiency: results of a systematic review. J Am Diet Assoc. 2008;108(5):832-9

13. Konstan MW, Pasta DJ, Wagener JS, VanDevanter DR, Morgan WJ. BMI fails to identify poor nutritional status in stunted children with CF. J Cyst Fibros. 2017:16(1):158-60.

14. Rizopoulos D. Joint models for longitudinal and time-to-event data : with applications in R. Boca Raton: CRC Press; 2012. xiv. p. 261

15. Andrinopoulou ER. Rizopoulos, D., Ruyun, J., Bogers, ad J.J.C., Lesaffre, E., Takkenberg, J.J.M. an introduction to mixed models and joint modeling: analysis of valve function over time. Ann Thorac Surg. 2012;93(6):1765-72.

16. Schluchter MD, Konstan MW, Davis PB. Jointly modelling the relationship between survival and pulmonary function in cystic fibrosis patients. Stat Med. 2002;21(9):1271-87.

17. Piccorelli AV, Schluchter MD. Jointly modeling the relationship between longitudinal and survival data subject to left truncation with applications to cystic fibrosis. Stat Med. 2012;31(29):3931-45.

18. Barrett J, Diggle P, Henderson R, Taylor-Robinson D. Joint modelling of repeated measurements and time-to-event outcomes: flexible model specification and exact likelihood inference. J R Stat Soc Series B Stat Methodol. 2015:77(1):131-48

19. Rizopoulos D. The R package JMbayes for fitting joint models for longitudinal and time-to-event data using MCMC. J Stat Softw. 2016:72(7):145.
20. Hickey GL, Philipson P, Jorgensen A, Kolamunnage-Dona R. Joint modelling of time-to-event and multivariate longitudinal outcomes: recent developments and issues. BMC Med Res Methodol. 2016;16(1):117

21. Knapp EA, Fink AK, Goss CH, Sewall A, Ostrenga J, Dowd C, et al. The Cystic Fibrosis Foundation patient registry. Design and methods of a National Observational Disease Registry. Ann Am Thorac Soc. 2016;13(7):1173-9.

22. Hankinson JL, Odencrantz JR, Fedan KB. Spirometric reference values from a sample of the general U.S. population. Am J Respir Crit Care Med. 1999; 159(1):179-87.

23. Wang $X$, Dockery DW, Wypij D, Fay ME, Ferris BG Jr. Pulmonary function between 6 and 18 years of age. Pediatr Pulmonol. 1993;15(2):75-88.

24. Szczesniak RD, Li D, Su W, Brokamp C, Pestian J, Seid M, et al. Phenotypes of rapid cystic fibrosis lung disease progression during adolescence and young adulthood. Am J Respir Crit Care Med. 2017;196:471-8.

25. Matloff N. Big-n versus Big-p in Big Data. In: Buhlmann P, Drineas P, Kane M, van der Laan M, editors. Handbook of big data. Boca Raton: CRC Press; 2016. p. 23-4.

26. Ferkol T, Rosenfeld M, Milla CE. Cystic fibrosis pulmonary exacerbations. J Pediatr. 2006;148(2):259-64

27. Shmueli G. To explain or predict? Stat Sci. 2010:25(3):289-310.

28. Chen $Y$, Wang $Y$. Variable selection for joint models of multivariate longitudinal measurements and event time data. Stat Med. 2017:36(24): 3820-9.

29. Andrinopoulou ER, Rizopoulos D. Bayesian shrinkage approach for a joint model of longitudinal and survival outcomes assuming different association structures. Stat Med. 2016;35(26):4813-23.

30. Neuhaus JM, McCulloch CE, Boylan RD. Analysis of longitudinal data from outcome-dependent visit processes: failure of proposed methods in realistic settings and potential improvements. Stat Med. 2018;37(29):4457-71.

\section{Publisher's Note}

Springer Nature remains neutral with regard to jurisdictional claims in published maps and institutional affiliations.

\section{Ready to submit your research? Choose BMC and benefit from:}

- fast, convenient online submission

- thorough peer review by experienced researchers in your field

- rapid publication on acceptance

- support for research data, including large and complex data types

- gold Open Access which fosters wider collaboration and increased citations

- maximum visibility for your research: over $100 \mathrm{M}$ website views per year

At BMC, research is always in progress.

Learn more biomedcentral.com/submissions 